\title{
A THEORY ON THE SLOPE OF THE IMF
}

\author{
H. Zinnecker \\ Max-Planck-Institut für Extraterr. Physik, Garching
}

Molecular clouds are clumpy, and the mass spectrum (dN/dM) of clumps scales with the clump mass $\mathrm{M}$ to the $\mathbf{- 1 . 5}$ power, as determined from $\mathrm{CO}$ observations of molecular clouds (Blitz 1988, Stutzki et al. 1989). The basic idea is to translate the mass spectrum of clumps into a mass spectrum of stars (that are assumed to form from these clumps) by virtue of a clump-star (i.e. initial-final) mass relation.

If the final, stellar mass $m$ scales with the $p$-th power of the initial clump mass $M$, one finds that the stellar mass spectrum $(\mathrm{dN} / \mathrm{dm})$ is a power law with index $\mathrm{x}=(\mathrm{p}+0.5) / \mathrm{p}$; for example, for $p=0.4$ one obtains $x=2.25$, very close to the index of the Salpeter IMF.

The physical justification for the power $p$ in the final-initial mass relation comes from the theory of ambipolar diffusion (for low-mass stars) and from the theory of radiation pressure acting on dust grains (for high mass atars): magnetic fields or radiation pressure prevent complete accretion of the clump mass onto a protostellar core and induce a clump-mass dependent inefficiency factor in the accretion process. Fragmentation of the clumps into smaller masses is ignored in this simplified picture, but could be incorporated.

There are several predictions following from this concept, among them that triggered star formation occurring in starburst galaxies should lead to an IMF directly reflecting the clump mass spectrum, since the magnetic and radiative forces against protostellar accretion are overwhelmed by pressure-induced or radiatively-induced implosion, causing very efficient accretion $(p=1)$ and thus a rather flat IMF.

\section{S266: A DISTANT HII REGION IN THE GALAXY}

\author{
A. Manchado, C. Esteban, J.M. Vilchez \\ Instituto de Astrofísica de Canarias, La Laguna, Tenerife, Spain
}

Long slit high and low resolution spectroscopy of S266 was conducted in order to investigate its precise nature. The $H_{\alpha}$ high resolution profile shows two different components; the narrow one extends all over the nebula while a very broad $\left(\Delta V_{F W H M}=700 \mathrm{~km} \mathrm{~s} \mathrm{~s}^{-1}\right)$ indicates that a strong wind is associated with the central star. From the narrow component we have worked out radial velocity $\left(V_{L R S}=23 \mathrm{~km} \mathrm{~s}^{-1}\right)$ which yields to a kinematic distance of a $9 \mathrm{kpc}$. This value implies a galactocentric distance of $19 \mathrm{kpc}$ which makes this region one of the most distant in the Galaxy.

The location of the observed lines ratios in a diagnostic diagram indicates that the object is an HII region class nebula. From the existing photometric data we found that the exciting star appears to be a late B supergiant. 